\title{
IMC Based Fractional Order Controller for Three Interacting Tank Process
}

\author{
Abdul Wahid Nasir ${ }^{1}$, Idamakanti Kasireddy ${ }^{2}$, Arun Kumar Singh ${ }^{3}$ \\ ${ }^{1,2,3}$ Department of Electrical and Electronics Engineering, NIT Jamshedpur, India \\ ${ }^{*}$ Corresponding author, e-mail: 2014rsee003@nitjsr.ac.in
}

\begin{abstract}
In model based control, performance of the controlled plant considerably depends on the accuracy of real plant being modelled. In present work, an attempt has been made to design Internal Model Control (IMC), for three interacting tank process for liquid level control. To avoid complexities in controller design, the third order three interacting tank process is modelled to First Order Plus Dead Time (FOPDT) model. Exploiting the admirable features of Fractional Calculus, the higher order model is also modelled to Fractional Order First Order Plus Dead Time (FO-FOPDT) model, which further reduces the modelling error. Moving to control section, different IMC schemes have been proposed based on the order of filter. Various simulations have been performed to show the greatness of Fractional order modelled system \& fractional order filters over conventional integer order modelled system \& integer order filters respectively. Both for parameters estimation of reduced order model and filter tuning, Genetic Algorithm $(G A)$ is being applied.
\end{abstract}

Keywords: Internal Model Control, Fractional Order Control, Interacting Tank Process, Model Order Reduction, Genetic Algorithm

Copyright $@ 2017$ Universitas Ahmad Dahlan. All rights reserved.

\section{Introduction}

The liquid level measurement and control are of critical importance for chemical process industries and the safety of the equipment they use. The control problem for such level process depends on how these tanks are coupled. Sometimes the tanks are so inter-connected that their level interacts, i.e. the level of one tank affects the dynamics of another tanks and vice-versa, a case of interacting process. It is undeniable fact that the level and flow control are the heart of chemical industries. In several such cases, e.g. petrochemical industries, pharmaceutical industries, food and beverages industries etc., level control problem of interacting tank arises. Thus the effective control of these variables shall prove very beneficent from economic point of view and also very much needed for the safety of equipments involved in the process $[1,2]$.

A wide range of different control strategies for level control such tank process has been proposed by different researchers based on conventional and soft computational techniques [35]. For the past couple of decades, the introduction of fractional order has resulted in improved performance both in modeling and control for similar control problems [6]. Fractional Calculus is actually the idea of extending the order of derivatives and integrals to non-integer orders [7]. These non-integer order fundamental operators, where ' $\alpha$ ' is the operator order and ' $a$ ' \& ' $t$ ' gives the limits of the operator is defined as follows:

$$
{ }_{a} D_{t}^{\alpha}= \begin{cases}d^{\alpha} / d t^{\alpha} & , \alpha>0 \\ 1 & , \alpha=0 \\ \int_{a}^{t}(d \tau)^{-\alpha} & , \alpha<0\end{cases}
$$

Different definitions of this fractional integro-differential is given by various experts namely Riemann-Liouville, Caputo, Grunwald-Lentnikov etc. [8]. Therefore, conventional integer order 
calculus or simply calculus can be thought as special case of this Fractional calculus. Due to this peculiar feature, Fractional calculus is making its presence felt in the field of science and technology in recent decades. Nowadays control engineers are exploiting Fractional Calculus in control theory basics like system modelling [9, 10], analysis [11, 12], and design [13, 14].

In present work, for the level control of three interacting tank process, Internal Model Control (IMC), a model based procedure has been engaged, where a process model is "embedded" in the controller [15]. As the process contains three storage elements, hence it is a $3^{\text {rd }}$ order system which comparatively makes the procedure of control design cumbersome. The order of this system is reduced to first order plus dead time (FOPDT) system, which in turn greatly reduce complexcities in controller design [16]. The adopted technique can also be extended to other higher order system like $4^{\text {th }}, 5^{\text {th }}$ and above. Taking a step further, the concept of Fractional Calculus is also being introduced in model reduction part, yielding a fractional order first order plus dead time (FO-FOPDT) system, thus by minimizing the modeling error.The main tuning parameters to tune in IMC are the filter time constants. But in the present work, the order of filter is considered as fraction entity, which increases the flexibility of the filter, thus affecting the performance of controlled system in a positive manner [17, 18]. Thus in overall modeling and control of the plant, closed loop control performance gets enhanced at two levels, first due to modeling and other includes the control design part.

The rest of the paper is organized as follows. IMC control scheme is discussed in brief in section 2. Basics of Genetic Algorithm (GA) which is used here as an optimization tool in obtaining reduced order model parameters and tunable controller parameters, given in section 3. Section 4, gives the mathematical modeling of a Three Tank Interacting Process, along with its linearization and model order reduction. Different schemes of proposed IMC are given in Section 5. The simulation results along with discussions are presented in Section 6. Based on the various results obtained conclusion is drawn and scope for future work is also given in Section 7.

\section{IMC Control Strategy}

The IMC technique was prominently used for chemical engineering applications in the past [19], which is considered to be a robust control method as well. The structure of IMC very much resembles the Smith Predictive controller [20]. Whenever the process encounters the unmeasured disturbances or control process uncertainty, IMC most of the times emerge to be very efficient in reducing the dynamic or static errors to ensure the robustness of the overall closed loop control system [21]. It incorporates the replica of the original process called "internal model". This internal model is connected in parallel with the original system to generate modified error signal for the controller in which inverse of the process model is embedded. Fulfilling the required criteria for being inverse of the process model to be stable, all right hand side zeros along with time delay if any of the original model are being factored out.

As shown in Figure 1, same control signal $u(s)$ is fed to the both original process $G p(s)$ and its model $\hat{G} p(s)$. Here $G p(s)$ is the original $3^{\text {rd }}$ order transfer function representation of three tank interacting process and $\hat{G p}(\mathrm{~s})$ is the reduced first order plus dead time model (FOPDT). It can be seen that output of $G p(s)$ and $\hat{G} p(s)$ is being used to generate an error signal ê(s), which gives the information that how much process model is deviating from the original process. Hence this information is used as feedback signal to generate the trimmed set point, by subtracting ê(s) from $r(s)$. Theoretically ê(s) can be zero or nullified when the model perfectly replicates every dynamics of the process, but practically it is not possible. The $q(s)$ is the controller transfer function which is defined as:

$$
q(s)=\hat{G}_{p}^{-}(s) \cdot f(s)
$$

where $\hat{G}_{p}{ }^{-}(s)$ is the invertible portion of the system. And $f(s)$ is the low pass filter making $q(s)$ proper or realizable which also possesses tunable parameters. 


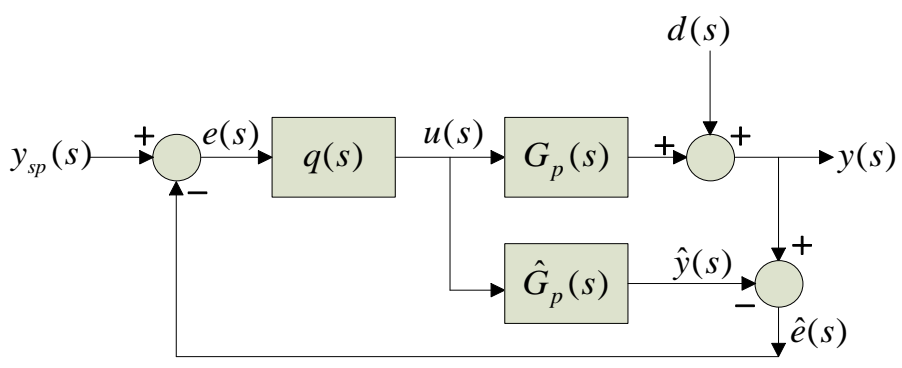

Figure 1. IMC Strategy

\section{Genetic Algorithm}

The use of genetic algorithms (GA) has become very common for various problem solving [22-24]. Since nowadays, cheap and fast speed computers are available which makes the application of GA feasible as it includes lots of complex calculations. The fundamental elements of GA were first proposed by Holland [25]. Later on, other literatures were reported [26-28] discussing its remarkable concept and applications. It is basically a nature inspired stochastic search algorithm based on natural evolution and selection which favours the fact that stronger individuals bear the greater potential to strive against all odds in a competing environment and the weaker one gradually extinct. A random set of parameters is being presumed to be the potential solution of the problem. These random parameters resemble the genes of chromosomes. Another parameter, a positive value commonly known as fitness function gives the measurement of "goodness" of the chromosomes for solving the problem and is somehow primarily depends on its objective function [29].

In many cases of controller designs, some parameters are required to be tuned optimally for the enhancement of the overall performance of control system [30-32]. In the present work, the parameter to be optimised is $\lambda$ (time constant of the filter) for integer order filter and if the filter is fractional in nature, then two tuning parameters i.e. $\lambda$ and order of the filter, ' $b$ ' are to be optimised. Although, Integral of Squared Error (ISE) is generally used as performance index for optimisation in control system design, but here Integral of Time weighted Squared Error (ITSE) has been considered because the later one gives less settling time [33]. The objective function to be minimised to obtain the value of controller parameter(s) for IMC is defined as:

$$
\text { ITSE }=\int_{0}^{\infty}\left\{y_{s p}(t)-y(t)\right\}^{2} \cdot t \cdot d t=\int_{0}^{\infty}\{e(t)\}^{2} \cdot t . d t
$$

The implementation of GA for the current problem can be well understood with the help following Figure 2.

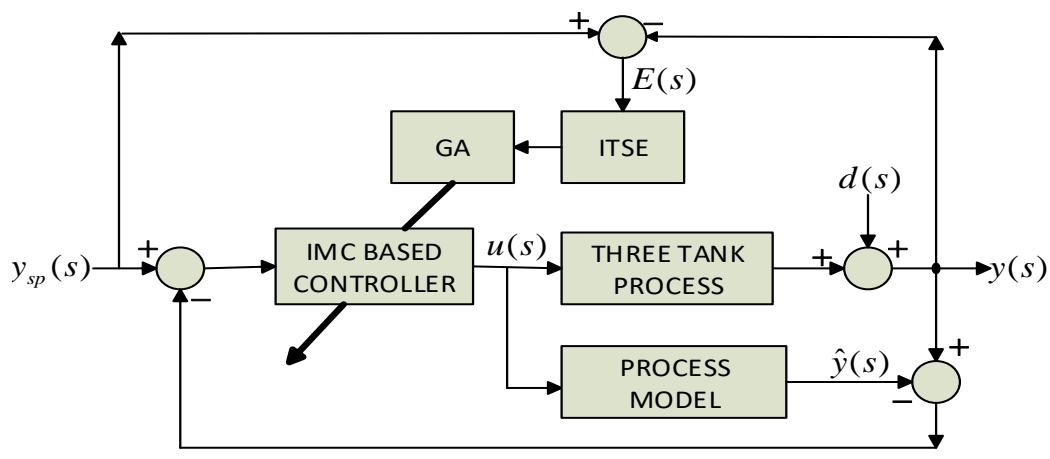

Figure 2. Block Diagram of Optimised IMC using GA 
Table 1 shows the GA characteristics used to obtain the required results.

Table 1. Selected Parameters of GA

\begin{tabular}{cc}
\hline Population Type & Double Vector \\
\hline Population Size & 50 \\
Fitness Scaling Function & Rank \\
Crossover Function & 0.8 \\
Crossover Fraction & Scattered \\
Migration Fraction & 0.2 \\
Ending Criterion & 100 Iterations \\
\hline
\end{tabular}

\section{Mathematical Modelling of Interacting Three Tank Liquid Level Process}

Consider the system shown in Figure 3, represents a benchmark problem which proves to be very effective in understanding multi tank level control strategy for interacting process. It consists of three identical cylindrical tanks Tank 1, Tank 2 and Tank 3 , having same cross sectional area, $A$. Tank 1 and Tank 2 are inter-connected at the bottom through a manual control valve, having valve coefficient $\beta_{12}$, using pipe of cross-sectional area $\alpha_{1}$. Similarly Tank 2 and Tank 3 are inter-connected through another manual control valve, having valve coefficient $\beta_{23}$, using pipe of cross-sectional area $\alpha_{2}$. Also there is one drain outlet regulated through a manual control valve, having valve coefficient $\beta 3$ using pipe of cross-sectional area $\alpha_{3}$. The pump helps the liquid flow to the Tank1 from sump through a control valve. As the liquid enters the Tank 1, some part of it also flows to Tank 2 and Tank 3 in accordance to process dynamics, resulting in rise of liquid levels of all these three tanks.

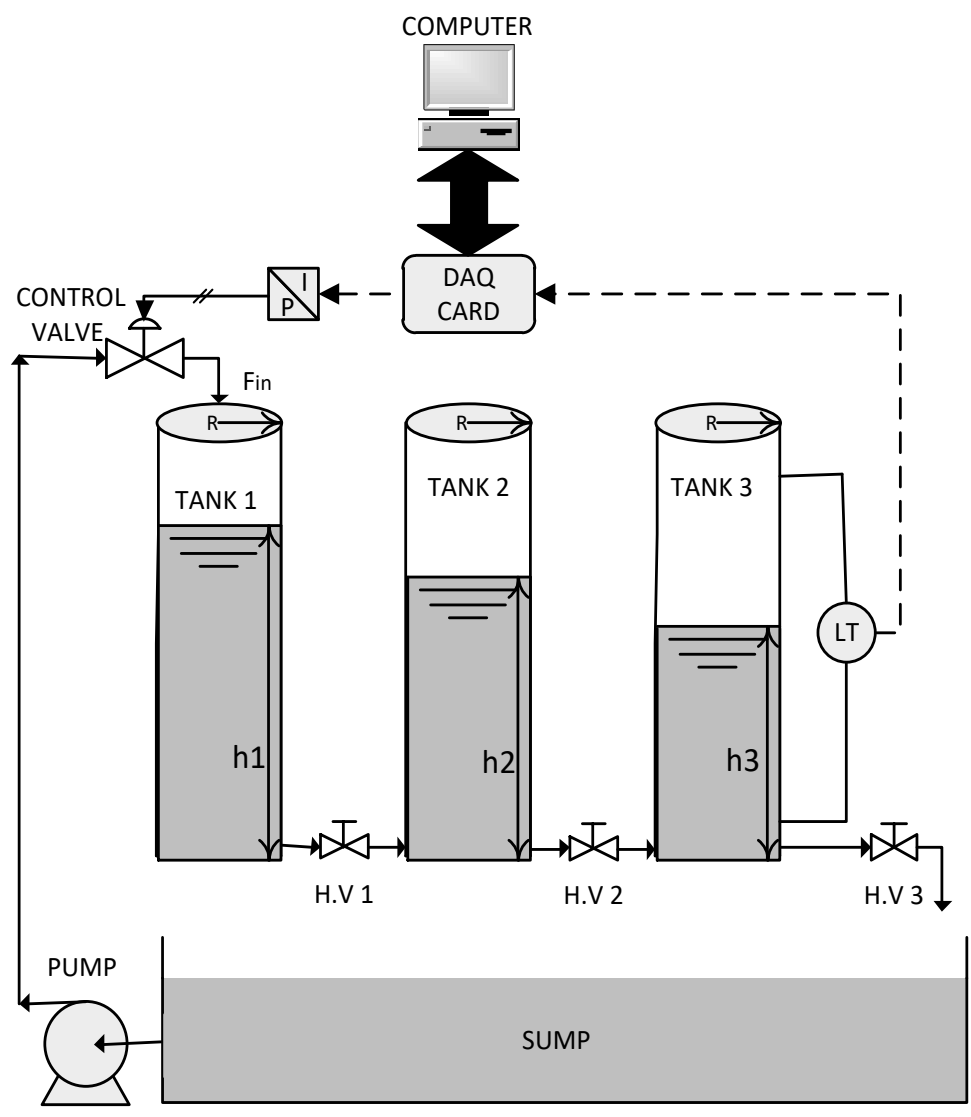

Figure 3. Three Interacting Tank Process 
The control problem considered here is to maintain the liquid level of Tank $3, h_{3}$ to desired value by controlling the fluid flow to Tank 1, Fin. Therefore, it is a SISO (Single Input Single Output) system with Fin being manipulated variable and $h_{3}$ being controlled variable. Assuming the fluid to be incompressible, the differential equations governing the dynamics of the plants based on conservation of mass are given below:

$$
\begin{aligned}
& \frac{d h_{1}(t)}{d t}=-\frac{\beta_{12} \alpha_{12}}{A_{1}} \sqrt{2 g\left(h_{1}(t)-h_{2}(t)\right)}+\frac{F_{i n}}{A_{1}} \\
& \frac{d h_{2}(t)}{d t}=\frac{\beta_{12} \alpha_{12}}{A_{2}} \sqrt{2 g\left(h_{1}(t)-h_{2}(t)\right)}-\frac{\beta_{23} \alpha_{23}}{A_{2}} \sqrt{2 g\left(h_{2}(t)-h_{3}(t)\right)} \\
& \frac{d h_{3}(t)}{d t}=\frac{\beta_{23} \alpha_{23}}{A_{3}} \sqrt{2 g\left(h_{2}(t)-h_{3}(t)\right)}-\frac{\beta_{3} \alpha_{3}}{A_{3}} \sqrt{2 g h_{3}(t)}
\end{aligned}
$$

where,

$h_{1}, h_{2}, h_{3}$ are the liquid levels of Tank 1 , Tank 2 and Tank 3 respectively,

$A_{1}=A_{2}=A_{3}=A=$ cross-sectional area of the cylindrical tank $=615 \mathrm{~cm}^{2}$,

$\alpha_{1}=\alpha_{2}=\alpha_{3}=\alpha=$ cross-sectional area of the pipe connecting tanks $=5 \mathrm{~cm}^{2}$,

$\beta_{12}=$ valve ratio of the valve connecting Tank 1 and Tank $2=0.9$,

$\beta_{23}=$ valve ratio of the valve connecting Tank 2 and Tank $3=0.8$,

$\beta_{3}=$ valve ratio of the valve connecting Tank 2 and Tank $3=0.3$,

$\mathrm{g}=$ acceleration due to gravity.

\subsection{Linearization}

Since the nature of plant is non-linear, therefore it requires linearization for controller design and implementation. For this purpose, the concept of Taylor's series is employed [34]. The chosen operating point is chosen as: Fin $=88 \mathrm{~cm}^{3} / \mathrm{sec}$ and $\mathrm{h}_{3}=3.5 \mathrm{~cm}$. Equation (7) gives the values of the state space matrix $A, B, C \& D$ and hence its corresponding transfer function is given by equation (8).

$$
\begin{aligned}
& A=\left[\begin{array}{ccc}
-0.3669 & 0.3669 & 0 \\
0.3669 & -0.6567 & 0.2829 \\
0 & 0.2829 & -0.3306
\end{array}\right] \\
& B=\left[\begin{array}{c}
1 / 615 \\
0 \\
0
\end{array}\right] \\
& C=\left[\begin{array}{lll}
0 & 0 & 1
\end{array}\right] \\
& D=0 \\
& T F=\frac{0.000173}{s^{3}+1.354 s^{2}+0.3627 s+0.00507}
\end{aligned}
$$

\subsection{Model Order Reduction}

Many papers have elaborated the concept of model order reduction and its application in control system [16, 35]. Since the process under study, is a third order system. To enhance computational performance and to bring ease in analysis and controller design, the original third 
order models are reduced to their corresponding First Order Plus Dead Time (FOPDT) and Fractional Order First Order Plus Dead Time (FO-FOPDT) for all the regions [37]. Here GA is used to determine the value of Gain $(K)$, Time Constant $(\tau)$ and delay $(L)$ to minimize the value of root mean square error (RMSE), the objective function considered here. Similarly, the unknown parameters of FO-FOPDT are obtained i.e. $\alpha$, the order of the filter, along with $\mathrm{K}$, $\mathrm{T}$ \& L. The general form of First Order Plus Dead Time (FOPDT) model, Fractional Order First Order Plus Dead Time (FO-FOPDT) model and the objective function RMSE is given by equation (9), equation (10) respectively.

$$
\begin{aligned}
& G_{F O P D T}=\frac{K}{\tau s+1} e^{-L s} \\
& G_{F O-F O P D T}=\frac{K}{\tau s^{\alpha}+1} e^{-L s}
\end{aligned}
$$

Step error minimization technique based on Genetic Algorithm (GA), which is discussed in the following subsection is employed to estimate the optimal value of model parameters. An identical step input is applied to both original third order system and its reduced order model, whose output responses are compared to generate error. This error is used to construct the objective function, which is taken to be root mean of squared error (RMSE) at present given by (11), $\mathrm{N}$ being the number of observations. This RMSE is being minimized using GA, which is illustrated in Figure 4.

$$
R M S E=\sqrt{\frac{\left(Y_{3 r d}-Y_{\text {reduced }}\right)^{2}}{N}}
$$

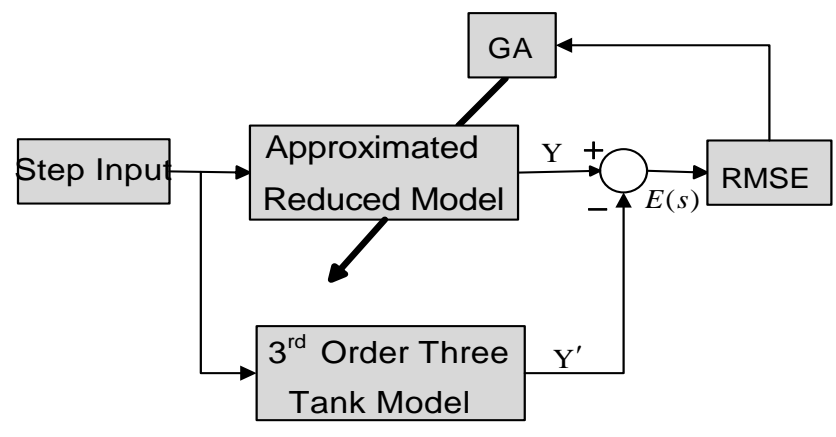

Figure 4. Lower Order Model Approximation using GA

\section{Different Schemes of Control Based on Order}

As already stated previously, reduced order model of the three interacting tank process will be used for the IMC filter. Since there are two types of reduced models available, first being Integer Order First Order Plus Dead Time (IO-FOPDT) model and other Fractional Order First Order Plus Dead Time (FO-FOPDT) model. Also in the present work, two categories of filter have been considered depending on the nature of its order. First one is the Integer Order filter, where the time constant $(\lambda)$ of the filter is the tunable parameter. And the second one is the Fractional Order filter, where the time constant $(\lambda)$ and the order of the filter $(b)$ are the two tunable parameters. Making use of the combinations of different reduced order models and filters, four IMC strategy arise, which can be listed as: (i) IO filter with IO-FOPDT, (ii) FO filter with IO-FOPDT, (iii) IO filter with FO-FOPDT and (iv) FO filter with FO-FOPDT. Firstly, the load disturbance is considered to be nil i.e. $\mathrm{d}(\mathrm{s})=0$. The different filters defined here also have to follow some constraints. Basically these constraints are imposed on the time constant and order of the filter. One should select the proper range of filter time constant to trade-off between 
speed of response and system robustness. And the order of the filter is such chosen that the filter is realizable i.e. becomes proper. Table 2 give different schemes of filters along with their tunable parameters fulfilling different constraints.

Table 2. Different Schemes of IMC Strategy

\begin{tabular}{cccc}
\hline Different Schemes & Filter Transfer Function, $\mathrm{q}(\mathrm{s})$ & Cntroller Parameter(s) & Constraints \\
\hline Scheme 1 & $\frac{67.895 s+1}{0.034(\lambda s+1)}$ & $\lambda$ & $1 \leq \lambda \leq 5$ \\
Scheme 2 & $\frac{67.895 s+1}{0.034\left(\lambda s^{b}+1\right)}$ & $\lambda, \mathrm{b}$ & $1 \leq \lambda \leq 5$ \\
Scheme 3 & $\frac{72.55 s^{1.015}+1}{0.034\left(\lambda s^{1.015}+1\right)}$ & $\lambda$ & $1 \leq \mathrm{b} \leq 1.5$ \\
Scheme 4 & $\frac{72.55 s^{1.015}+1}{0.034\left(\lambda s^{b}+1\right)}$ & & $1 \leq \lambda \leq 5$ \\
& & $\lambda, \mathrm{b}$ & $1 \leq \lambda \leq 5$ \\
& & & $1.015 \leq \mathrm{b} \leq 1.5$ \\
\hline
\end{tabular}

Now the same set of controllers, as shown in Table 3 is implemented for the disturbance rejection, i.e. in these cases $d(s) \neq 0$. Generally, the nature of disturbance is assumed to be first order system, therefore following form of disturbance given by equation (12) has been considered and the results are shown in the next section.

$$
d(s)=g_{d}(s) r(s)
$$

where

$$
g_{d}(s)=\frac{1}{(10 s+1)}
$$

\section{Results and Discussions}

It has been already discussed, that model of the plant plays a vital role in current scheme of control strategy. Therefore, based on the concept fractional order model accurate model of the plant is obtained.. Hence the present section is divided into two parts. First part deals with the results obtained for modeling in reference to model order reduction, followed by second part where the controller performance is analyzed through various data and plots.

The $3^{\text {rd }}$ order transfer function given by (8), is approximated to lower order integer and fractional order model given by (9) \& (10) respectively, for bringing ease in controller design by implementing the methodology as discussed in Section 4.2. Hence the estimated transfer function for the approximated models along with their RMSE values is given in following Table 3. Figure 5 represents the step responses for $3^{\text {rd }}$ order model along with their approximated lower order integer and non-integer models respectively.Both time domain analysis given by Figure 5 data in Table 3, support in favor of fractional order modeling in cases where the reduction of model order is concerned.

Table 3. Model Order Reduction

\begin{tabular}{ccc}
\hline Nature of Model & Transfer Function & RMSE \\
\hline Integer Order & $\frac{0.034}{67.895 s+1} e^{-3.767 s}$ & 0.0095 \\
Fractional Order & $\frac{0.034}{72.55 s^{1.015}+1} e^{-3.19 s}$ & 0.0034 \\
\hline
\end{tabular}




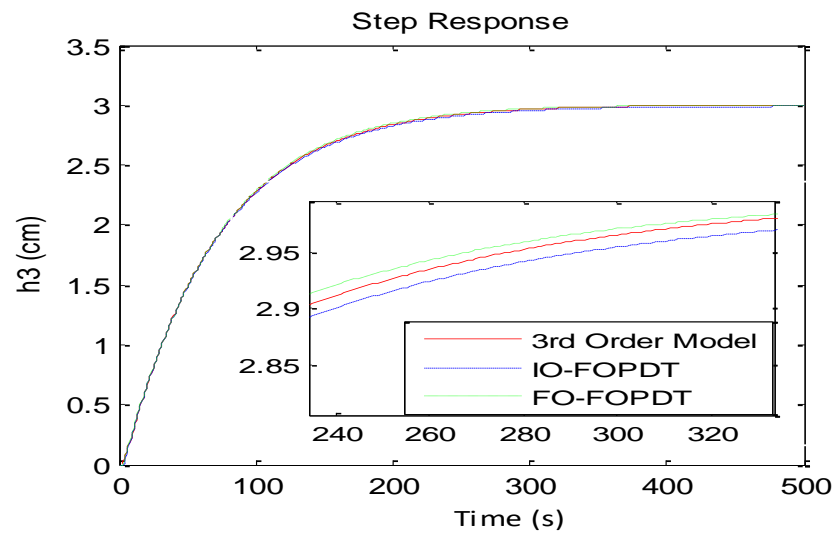

Figure 5. Open-Loop Step Response

Moving forward to control section, a series of MATLAB program is developed and simulated for finding the controller parameters for different IMC strategies. Since, ITSE is being minimized with the help of GA, to obtain the controller parameters for all four schemes i.e. Scheme 1, Scheme 2, Scheme 3 and Scheme 4. Table 4 shows the value of controller parameters along with the values of their corresponding performance index i.e. ITSE for each schemes. Figure 5 and Figure 6 represent the servo response for $d(s)=0$ and servo-regulatory response for $d(s) \neq 0$, when the disturbance of $20 \%$ is applied at time $t=100$ seconds, for all schemes respectively. These responses are having zoomed version of time response for a specific duration of time in the inset, so that the responses for each schemes can be identified easily. Again Fractional calculus when introduced in IMC filter design, further enhances the performance of the closed loop control system. Table 4 clearly indicates that the ITSE value is maximum for Scheme 1 and is gradually decreasing for Scheme 2, Scheme 3 with Scheme 4 being minimum, validating the supremacy of fractional order modeling and control over integer order.

Table 4. Controller Parameters with their Corresponding ITSE Value

\begin{tabular}{cccc}
\hline Different Schemes & Cntroller Parameter(s) & ITSE Value with $d(s)=0$ & ITSE Value with $d(s) \neq 0$ \\
\hline Scheme 1 & $\lambda=1.001$ & 25.75 & 348 \\
Scheme 2 & $\lambda=1 ; b=1.282$ & 23.34 & 289 \\
Scheme 3 & $\lambda=1$ & 21.99 & 281 \\
Scheme 4 & $\lambda=1 ; b=1.432$ & 19.91 & 213 \\
\hline
\end{tabular}

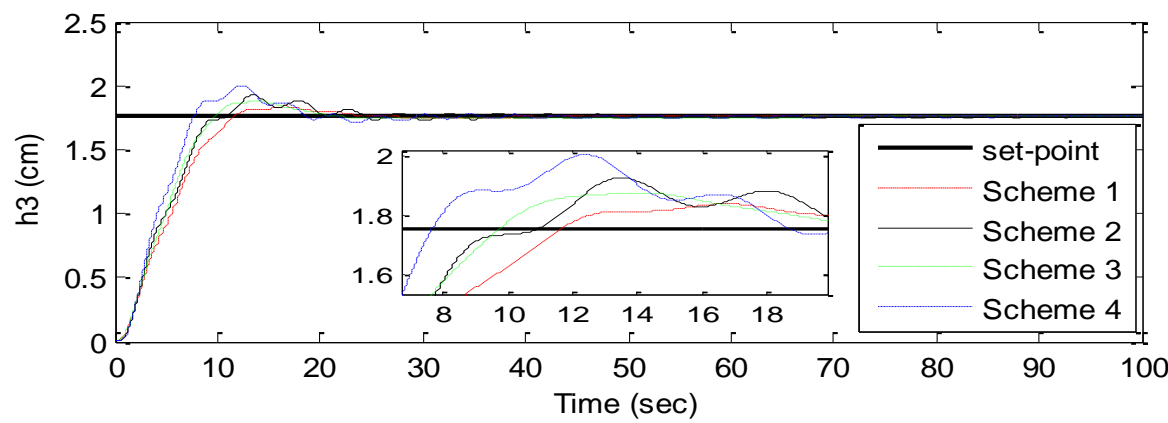

Figure 6. Servo Response 


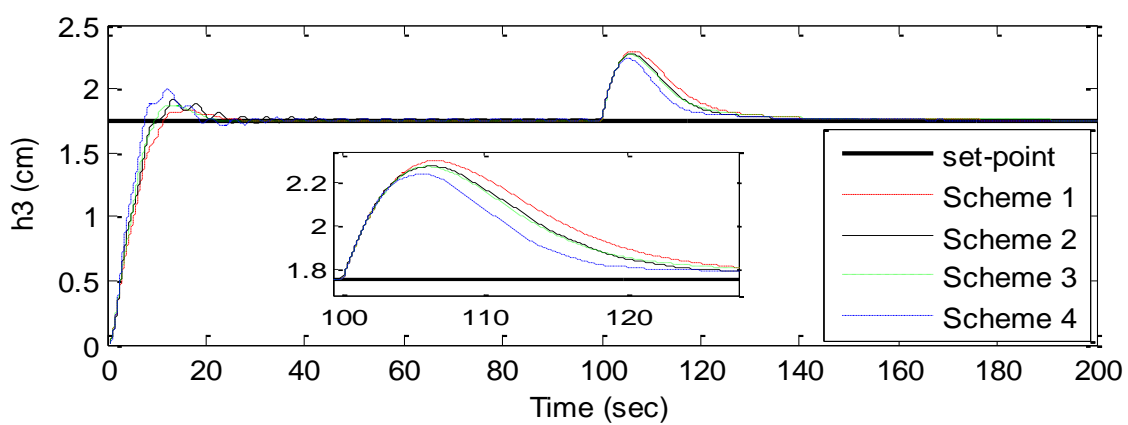

Figure 7. Servo - Regulatory Response

\section{Conclusions}

In this paper, IMC based fractional order controller has been reported for the level control of three interacting tank process. Four different schemes of IMC based controller arising from the combinations of different nature of system and IMC filter in terms of their order (Integer or Fractional), have been implemented for achieving the objective. The controller parameter(s) for all these four schemes are obtained using the same performance index, which is ITSE in the present case. After going through the simulated results, it can be concluded that that the inclusion of Fractional calculus not only reduce the modelling error but also enhances the control performance for IMC based control system. Also the designed control schemes very effectively meet the servo-regulatory performance. The methodology implemented here can be extended to any other model based control technique.

\section{References}

[1] Vijayan S and Avinashe KK. Soft Computing Technique and Conventional Controller for Conical Tank Level Control, Indonesian Journal of Electrical Engineering and Informatics, 2016; 4(1): 65-73.

[2] Chakravarthi MK and Venkatesan N.Experimental Validation of a Multi Model PI Controller for a Non Linear Hybrid System in LabVIEW, Telkomnika, 2015; 13(2): 547-555.

[3] Roy P and Roy BK. Fractional order PI control applied to level control in coupled two tank MIMO system with experimental validation, Control Engineering Practice; doi: 10.1016/j.conengprac.2016.01.002.

[4] Sadeghi MS, Safarinejadian B and Farughian A. Parallel distributed compensator design of tank level control based onfuzzy Takagi-Sugeno model, Applied Soft Computing, 2014; 21: 280-285.

[5] Taoyan Z, Ping LI and Jiangtao CAO. Study of Interval Type-2 Fuzzy Controller for the Twin-tank Water Level System, Chinese Journal of Chemical Engineering, 2012; 20(6): 1102-1106.

[6] Tepljakov A, Petlenkov E, Belikov J and Halás M. Design and Implementation of Fractional-order PID Controllers for a Fluid Tank System, 2013 American Control Conference; 2013: 177-1782.

[7] Oldham KB and Spanier J. The Fractional Calculus, Academic Press, New York, 1974.

[8] Monje CA, Chen Y, Vinagre BM, Xue D, and Feliu V. Fractional order systems and controls: Fundamental and applications. London: Springer-Verlag. 2010: 09-34.

[9] Lino P, Maione G, Saponaro F. Fractional Order Modeling of High-Pressure Fluid-Dynamic Flows: An Automotive Application, IFAC- Papers Online. 2015; 48(1): 382-387.

[10] Djamah T, Mansouri R, Djennoune S, Bettayeb M. Optimal low order model identification of fractional dynamic systems. Applied Mathematics and Computation. 2008; 206(2): 543-554.

[11] Chen YQ, Ahn HS, Podlubny I. Robust stability check of fractional order linear time invariant systems with interval uncertainties. Signal Processing. 2006; 86(10): 2611-2616.

[12] Li Y, Chen YQ, Podlubny I. Mittag-Leffler stability of fractional order nonlinear dynamic systems. Automatica. 2009; 45(8): 1965-1969.

[13] Pommier V, Sabatier J, Lanusse P, Oustaloup A. Crone control of a nonlinear hydraulic actuator. Control Engineering Practice. 2002; 10(4): 391-402.

[14] Chen C, HU L, Wang L, Gao S, Li C. Adaptive Particle Swarm Algorithm for Parameters Tuning of Fractional Order PID Controller. TELKOMNIKA. 2016; 14(2): 478-488.

[15] Wayne B. Process Control: Modeling, Design and Simulation. $2^{\text {nd }}$ Ed., Perintice Hall, 2002.

[16] Lavania S and Nagaria D. Evolutionary approach for model order reduction, Perspectives in Science, 2016; 8: 361-363. 
[17] Nasir AW, Singh AK. IMC Based Fractional Order Controller for Non-Minimum Phase System. Annual IEEE India Conference, INDICON. 2015: 1-6.

[18] Bettayeb M, Mansouri R. Fractional IMC-PID-filter controllers design for non integer order systems, Journal of Process Control, 2014; 24(4): 261-271.

[19] Morari M, Zafirion E. Robust Process Control, Englewood Cliffs, NJ: Prentice Hall, 1989.

[20] Wen WF, Rad AB. Fuzzy Adaptive Internal Model Control. IEEE Trans. Ind. Electron. 2012; 47(1): 193-202.

[21] Xia C, Yan Y, Song P, Shi T. Voltage Disturbance rejection for matrix converted based PMSM drive System using Internal Model Control. IEEE Trans. Ind. Electron. 2000; 59(1): 361-372.

[22] Shabbir F, Omenzetter P. Model updating using genetic algorithms with sequential niche technique. Engineering Structures. 2016; 120: 166-182.

[23] Jia Y, Yang X. Optimization of control parameters based on genetic algorithms for spacecraft altitude tracking with input constraints. Neurocomputing. 2016; 177: 334-341.

[24] Yanik S, Surer O, Oztaysi B. Designing sustainable energy regions using genetic algorithms and location-allocation approach. Energy. 2016; 97: 161-172.

[25] Holland JH. Adaption in Natural \& Artificial Systems. Cambridge MA: MIT Presss, 1975.

[26] Goldberg DE. Genetic Algorithms in search Optimization and Machine Learning. Addison Wesley 1989.Lawrence Davis, Handbook of Genetic Algorithms. New York. Van Nostrand Reinhold Company, 1991.

[27] Tahami F, Nademi H and Rezaei M. Maximum Torque per Ampere Control of Permanent Magnet Synchronous Motor Using Genetic Algorithm. TELKOMNIKA. 2011; 9(2): 237-244.

[28] Ronghui $\mathrm{H}$ and Xun $\mathrm{L}$ and Xin $\mathrm{Z}$ and Huikun P. Flight Reliability of Multi Rotor UAV Based on Genetic Algorithm. TELKOMNIKA. 2016; 14(2A): 231-240.

[29] Man KF, Tang TS, Kwong S. Genetic Algorithms: Concepts and Applications. IEEE Trans. Ind. Electron. 1996; 43(5): 519-534.

[30] Firdaus AR, Rahman AS. Genetic Algorithm of Sliding Mode Control Design for Manipulator Robot. TELKOMNIKA. 2012; 10(4): 645-654.

[31] Sundareswaran K, Devi V, Sankar S, Nayak PSR, Peddapati S. Feedback controller design for a boost converter through evolutionary algorithms. IET Power Electronics. 2014; 7(14): 903-913.

[32] Hasaneien HM, Muyeen SM. Design Optimization of Controller Parameters Used in Variable Speed WindEnergy Conversion System by Genetic Algorithms. IEEE Transaction in Sustainable Energy. 2012; 3(2): 200-208.

[33] Westcott JH. The Minimum Moment of Error Squared Criterion: A new performance criterion for servo mechanisms. IEEE Proc. Measurements Section. 1954: 471-480.

[34] Stephanopoulos G. Chemical Process Control: Chemical Process Control. $1^{\text {st }}$ Ed. Prentice Hall. 2012: 116-126.

[35] Seborg DE, Mellichamp DA, Edger TF. Process Dynamics and Control. $3^{\text {rd }}$ Ed., John Wiley and Sons. 2010: 102-103. 